\title{
Work-Focused Treatment of Common Mental Disorders and Return to Work: A Comparative Outcome Study
}

\author{
Suzanne E. Lagerveld \\ TNO Quality of Life/Work \& Employment Hoofddorp, \\ The Netherlands
}

\author{
Roland W. B. Blonk \\ TNO Quality of Life/Work \& Employment Hoofddorp, \\ The Netherlands and Utrecht University
}

\author{
Veerle Brenninkmeijer, Leoniek Wijngaards-de Meij, and Wilmar B. Schaufeli \\ Utrecht University
}

\begin{abstract}
The aim of this study was to compare the effectiveness of two individual-level psychotherapy interventions: (a) treatment as usual consisting of cognitive-behavioral therapy (CBT) and (b) work-focused CBT (W-CBT) that integrated work aspects early into the treatment. Both interventions were carried out by psychotherapists with employees on sick leave because of common mental disorders (depression, anxiety, or adjustment disorder). In a quasi-experimental design, 12-month follow-up data of 168 employees were collected. The CBT group consisted of 79 clients, the W-CBT group of 89. Outcome measures were duration until return to work (RTW), mental health problems, and costs to the employer. We found significant effects on duration until RTW in favor of the W-CBT group: full RTW occurred 65 days earlier. Partial RTW occurred 12 days earlier. A significant decrease in mental health problems was equally present in both conditions. The average financial advantage for the employer of an employee in the W-CBT group was estimated at \$5,275 U.S. dollars compared with the CBT group. These results show that through focusing more and earlier on work-related aspects and RTW, functional recovery in work can be substantially speeded up within a regular psychotherapeutic setting. This result was achieved without negative side effects on psychological complaints over the course of 1 year. Integrating work-related aspects into CBT is, therefore, a fruitful approach with benefits for employees and employers alike.
\end{abstract}

Keywords: return to work, mental disorder, cognitive-behavioral therapy, sickness absence, treatment outcome

In recent years, effective treatment of employees suffering from mental health problems has received increasing attention (Glozier, 2002; Goetzel, Ozminkowski, Sederer, \& Mark, 2002; Nieuwenhuijsen, 2004; Seymour \& Grove, 2005; Wang, Adair, \& Patten, 2006). This is especially the case for mental disorders with a high (and increasing) prevalence rate within the working population, such as depression, anxiety, and adjustment disorder (Andrea et al., 2004; Boedeker \& Klindworth, 2007). These disorders are also

This article was published Online First February 6, 2012.

Suzanne E. Lagerveld, TNO Quality of Life/Work \& Employment Hoofddorp, The Netherlands; Roland W. B. Blonk, TNO Quality of Life/ Work \& Employment Hoofddorp, The Netherlands and Department of Social and Organizational Psychology, Utrecht University, The Netherlands; Veerle Brenninkmeijer, Department of Social and Organizational Psychology, Utrecht University, The Netherlands; Leoniek Wijngaards- de Meij, Department of Methodology and Statistics, Utrecht University, The Netherlands; Wilmar B. Schaufeli, Department of Social and Organizational Psychology, Utrecht University, The Netherlands.

The authors wish to acknowledge the therapists and management of Ensis, especially Colin van der Heiden and Gerda Methorst, and Cora Maas for her statistical advise.

Correspondence concerning this article should be addressed to Suzanne E. Lagerveld, TNO Quality of Life/Work \& Employment, P.O. Box 718, 2130 AS Hoofddorp, The Netherlands. E-mail: suzanne.lagerveld@tno.nl called "common mental disorders" (Nieuwenhuijsen, 2004). Such disorders are associated with impaired work functioning and problems in work participation such as long-term sick leave (Kessler et al., 2006; Sanderson \& Andrews, 2006; Seymour \& Grove, 2005; van der Bossche \& Houtman, 2007).

Decreased work participation due to mental ill health is problematic as it leads to immense costs to employers, the "tax payer," and insurance companies (Goetzel, Hawkins, Ozminkowski, \& Wang, 2003; Greenberg et al., 2003; Seymour \& Grove, 2005; Stress Impact, 2006). Furthermore, decreased participation in work has direct effects on people's well-being. Those who are unable to participate in work lose a valuable source of social support and interpersonal contacts (Plaisier, 2009; Seymour \& Grove, 2005); they might lose part of their income and consequently tend to develop even more (severe) psychological symptoms (Price, van Ryn, \& Vinokur, 2002). Not surprisingly, work participation is rated by sick people as the third most important aspect of their quality of life (Bowling, 1995). Employees on sick leave with mental disorders would, therefore, benefit from interventions that enable them to return to work.

Although many interventions aimed at the reduction of mental health complaints have been developed and evaluated (e.g., Butler, Chapman, Forman, \& Beck, 2006), less scientific evidence is available on methods that successfully enhance return to work (RTW) for workers with common mental health disorders (Nieu- 
wenhuijsen et al., 2008; Rebergen, 2009). In particular, the effectiveness of psychotherapeutic interventions on RTW is largely unknown, while this type of intervention is often delivered to workers with common mental health problems (Kessler et al., 2003; Stress Impact, 2006; ten Have, de Graaf, Vollebergh, \& Beekman, 2004). The findings of the few studies in which psychotherapeutic interventions (in most cases CBT) have been evaluated, show that they were equally or less effective in enhancing RTW compared with other interventions, such as CBT-based guidance provided by occupational physicians (Blonk, Brenninkmeijer, Lagerveld, \& Houtman, 2006; de Vente, Kamphuis, Emmelkamp, \& Blonk, 2008; Lander, Firche, Tornemand, Andersen, \& Kirkeskov, 2009; Nystuen, Hagen, \& Herrin, 2006; Rebergen, 2009).

The small number of controlled studies on the effects of psychotherapy on RTW to date underlines the need for more research in this field, and also the necessity of adapting existing psychotherapeutic interventions. There are some indications that workdirected interventions in combination with cognitive-behavioral therapy (CBT) components are effective with respect to RTW for those absent with common mental health problems (Blonk et al., 2006; Schene, Koeter, Kikkert, Swinkels, \& McCrone, 2007; van der Klink, Blonk, Schene, \& van Dijk, 2003; and for a review see Rebergen, 2009). For employees absent with musculoskeletal disorders (including low back pain) such multimodal approaches (that combine interventions on health condition and work) have also been proven to stimulate RTW (Anema et al., 2007; Arnetz, Sjögren, Rydéhn, \& Meisel, 2003; Durand \& Loisel, 2001; Lambeek, van Mechelen, Knol, Loisel, \& Anema, 2010;Loisel et al., 1997).

In regular CBT, offered by mental health professionals, a focus on work and RTW is often lacking (Keuzenkamp, Kok, \& van Seters, 2002; Kidd, Boyd, Bieling, Pike, \& Kazarian-Keith, 2008; Rebergen, 2009). The effectiveness of psychotherapy on RTW may, therefore, be enhanced when work (or RTW) is more explicitly addressed during treatment and psychologists (CBT experts) are trained in workplace issues. The current study evaluates the effectiveness of work-focused CBT provided by psychotherapists with regard to RTW for employees on sick leave owing to common mental health problems.

The work-focused CBT intervention (W-CBT) employs the same conceptual framework as is used for regular CBT, which is largely based on the work of Beck (1976). In short, CBT theory states that dysfunctional (coping) behavior and mental health symptoms are not merely the consequence of a stressful situation (e.g., work pressure), but that the appraisal of this situation (cognition) plays a crucial role. CBT can be used to intervene in any of these three components (situation, behavior, and cognitions) from both a cognitive and a behavioral perspective. The relationship between cognitive change and behavior change is complex and interactive, with change in one domain promoting change in the other, and vice versa (Meichenbaum \& Cameron, 1982; Wright, 2006). Two main intervention approaches within CBT can be distinguished, targeting a change of "dysfunctional cognitions" and acquiring of effective coping skills, for example, by following a graded activity principle (Beck, Rush, Shaw, \& Emery, 1979; Butler et al., 2006; Ellis, 1997; Hobbis \& Sutton, 2005; Longmore \& Worrell, 2007).

The central idea behind the work-focused intervention is that any CBT technique may be applied to the work context, in order to achieve regular psychotherapy treatment goals and RTW. We expected that CBT techniques applied to the work context might change a stressful work situation (e.g., the client might decide to ask for a transfer to another department), change the appraisal of work stressors (e.g., the client may feel less incompetent when the supervisor is not satisfied with his work), or change the dysfunctional behavior itself (e.g., stimulating gradual RTW as a form of behavioral activation). Hence, integrating (return to) work aspects would contribute to a change in dysfunctional work-related behaviors or symptoms, thereby facilitating RTW.

In W-CBT special attention was given to gradual exposure to the workplace because this element was included in two previous CBT-based interventions (Blonk et al., 2006; van der Klink et al., 2003) that proved to be effective in RTW. Following graded activity principles, we assumed that work participation could best be stimulated by gradually exposing patients to the work setting. Gradual work resumption can help individuals develop more effective coping skills to deal with (return to) work-related stressors. According to several authors (e.g., Blonk et al., 2006; van der Klink et al., 2003) these coping skills can best be acquired and strengthened gradually within the workplace itself. For instance, Blonk and colleagues describe how gradual work resumption may foster experiences of success. That is:

By performing the tasks individuals are able to do (with respect to duration, intensity or complexity for instance) individuals may acquire a sense of self-efficacy and control. Exposure to work may also provide experiences that challenge dysfunctional beliefs. The correction of dysfunctional beliefs is viewed as one of the mechanisms that can explain the effectiveness of exposure (Blonk et al., 2006, p 131).

In sum, stimulating partial RTW may be an effective method to stimulate effective coping, secure success experiences at work, and challenge dysfunctional beliefs, thereby promoting full RTW (see also Noordik, van Dijk, Nieuwenhuijsen, \& van der Klink, 2009; van Rhenen, Schaufeli, van Dijk, \& Blonk, 2008).

The aim of the current study was to evaluate the effectiveness of CBT with additional integrated modules addressing work and return to work (W-CBT) compared with regular psychotherapy (in this case CBT). The empirical evidence and a priori explanations for the effectiveness of work-focused CBT described above gave rise to the following hypotheses.

Hypothesis 1a: Work-focused cognitive-behavioral therapy (W-CBT) will be more effective with respect to RTW compared with regular CBT.

Hypothesis 1b: Because of its effectiveness in RTW, W-CBT will, from an employers' perspective, be economically superior compared with regular CBT.

The effectiveness of CBT in improving mental health has been widely demonstrated in controlled trials for a variety of disorders including mood and anxiety disorders (Butler et al., 2006). Various studies in which CBT-based interventions were evaluated among sick-listed workers with common mental health problems have shown that symptoms decrease over time, irrespective of treatment content (see, e.g., Blonk et al., 2006; Nieuwenhuijsen, 2004; Schene et al., 2007; van der Klink et al., 2003). It was, therefore, expected that the current two types of CBT would not differ in 
mental health outcomes. This gave rise to the following hypothesis on mental health.

Hypothesis 2: Work-focused cognitive-behavioral therapy (W-CBT) will be equally effective with respect to improving mental health compared with regular CBT.

\section{Method}

\section{Participants}

Employees on sick leave $(100 \%$ absent at the onset of their absenteeism and not fully returned to work at the start of treatment) due to psychological problems (common mental health disorders) were recruited to participate in the study by clinical therapists from an outpatient mental health center. These "common mental health" disorders include one or more of the following diagnoses according to DSM-IV criteria (APA, 1994): Adjustment Disorder, Undifferentiated Somatoform Disorder, Anxiety Disorder (Posttraumatic Stress Disorder was excluded), or a Mood Disorder (Major Depressive Disorder was excluded). From an ethical perspective, we excluded those with more severe mental disorders at this point, until the effectiveness of W-CBT could be established for the less severe groups.

As depicted in Figure 1, 208 out of 250 eligible clients agreed to participate. A total of 168 clients filled out the first questionnaire (response rate 67\%). The main reason for nonparticipation was "the burden of filling out questionnaires." One person reported conflict at work. Participants worked in a variety of jobs, such as administrative jobs (13\%), commercial service jobs (19\%), health care $(20 \%)$, education $(6 \%)$, trade $(6 \%)$, construction $(5 \%)$, civil services $(5 \%)$, and transport $(3 \%)$. In addition, the companies in which the participants were employed varied in size: $30 \%$ worked in companies with fewer than 30 employees, $29 \%$ in companies with $30-100$ employees, and $41 \%$ in companies with over 100 employees. Other baseline characteristics of our sample and the duration of the treatment participants received are presented in Table 1.

\section{Procedure}

During intake at the mental health center, employees were screened for inclusion criteria (see above) by a clinical therapist. When a client met these inclusion criteria, the therapist would briefly describe the study and hand over an information brochure about the research project. Upon acceptance, approval of the proposed treatment plan, and signing of an informed consent, the baseline questionnaire was sent to the client per mail. Treatment sessions generally started 1 week after the first questionnaire was filled out. Follow-up questionnaires were sent at fixed times: 1, 3, 6,9 , and 12 months after baseline. Clients who did not return the 1-year follow-up questionnaire were contacted by telephone in order to retrieve the follow-up RTW data.

\section{Design}

In a quasi-experimental design, four nearby departments of a mental health center in a large urban area recruited participants for this study. Each department employed about 15 psychotherapists. The therapists who participated in this study were recruited by their management team, and all therapists attended a meeting organized by the research team at the start of the project, in which the rationale for and procedure of the trial were explained.

To avoid contamination between treatment groups, the departments were assigned to perform either regular cognitivebehavioral therapy (CBT, 2 departments) or CBT according to the work-focused protocol (W-CBT, 2 departments). Registration and allocation of clients to a specific therapist occurred centrally for all departments. This process did not involve content-driven choices on the part of either the therapist or the client. Clients were

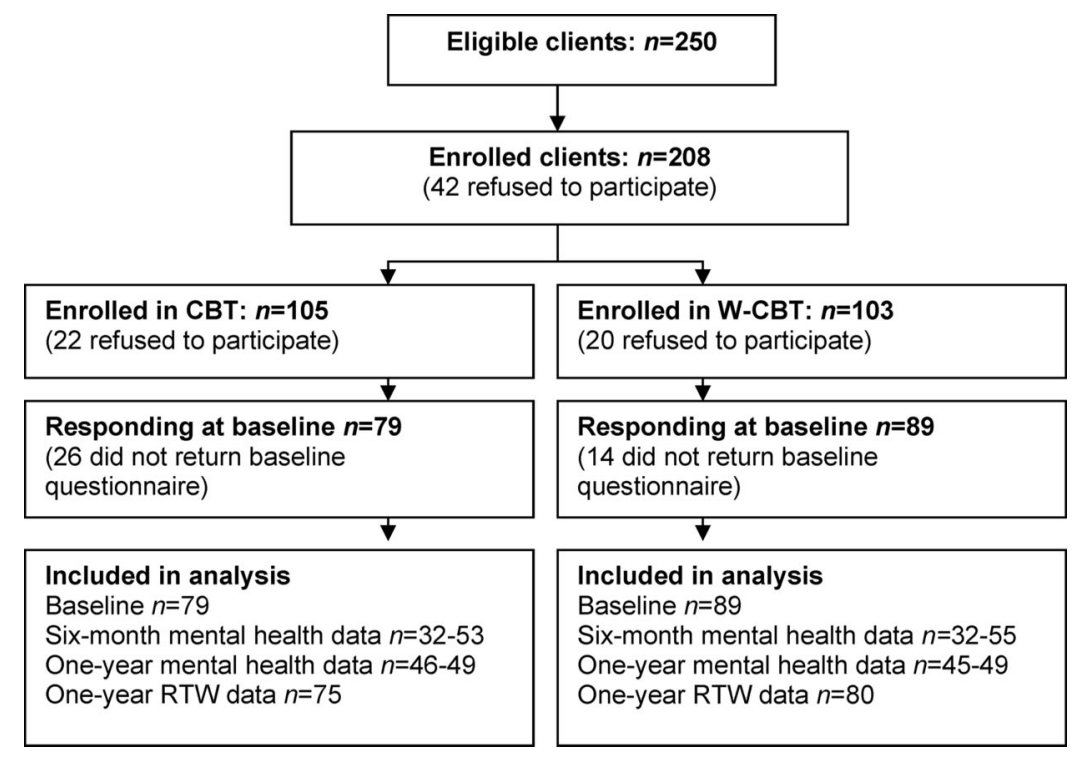

Figure 1. Flowchart participant recruitment. $\mathrm{CBT}=$ Cognitive Behavioral Therapy; W-CBT $=$ Work-focused Cognitive Behavioral Therapy. 
Table 1

Baseline Characteristics of Participants and Treatment Duration

\begin{tabular}{lccc}
\hline & $\begin{array}{c}\text { CBT group } \\
(n=79)\end{array}$ & $\begin{array}{c}\text { W-CBT group } \\
(n=89)\end{array}$ & $\begin{array}{c}\text { Total } \\
(n=168)\end{array}$ \\
\hline Demographics (client characteristics) & & & \\
Mean age (SD) & $41.3(10.4)$ & $40.2(9.6)$ & $40.7(9.9)$ \\
Gender (\% female) & $67 \%$ & $54 \%$ & $60 \%$ \\
Married or cohabiting & $67 \%$ & $86 \%$ & $77 \%$ \\
Lower vocational/general secondary education & $37 \%$ & $37 \%$ & $37 \%$ \\
Intermediate vocational education & $27 \%$ & $35 \%$ & $31 \%$ \\
Higher education & $36 \%$ & $27 \%$ & $31 \%$ \\
Disorder \& Treatment characteristics & & & \\
Adjustment disorder (MHC diagnosis) & $62 \%$ & $72 \%$ & $67 \%$ \\
Anxiety (MHC diagnosis) & $15 \%$ & $12 \%$ & $13 \%$ \\
Depression (MHC diagnosis) & $18 \%$ & $16 \%$ & $17 \%$ \\
Other mental disorder (MHC diagnosis) & $5 \%$ & - & $2,4 \%$ \\
Mean DASS stress score (SD) & $12.8(4.9)$ & $11.42(4.6)$ & $12.1(4.8)$ \\
Mean SCL90 depression score (SD) & $40.1(11.8)$ & $38.9(10.4)$ & $39.4(11.0)$ \\
Mean SCL90 anxiety score (SD) & $21.9(8.3)$ & $22.5(8.3)$ & $27.9(10.6)$ \\
Mean MBI emotional exhaustion score (SD) & $3.5(1.7)$ & $3.3(1.8)$ & $3.4(1.8)$ \\
Mean weeks on waiting list of MHC (SD) & $4.4(2.6)$ & $5.9(2.9)$ & $5.2(2.9)$ \\
Mean number of therapeutic sessions & $11.4(3.6)$ & $11.1(3.7)$ & $11.2(3.6)$ \\
Mean months in therapy & $5.7(2.6)$ & $5.5(2.9)$ & $5.6(2.7)$ \\
Work Characteristics & & & \\
Mean weekly working hours by contract (SD) & $33.7(6.6)$ & $33.2(7.9)$ & $33.4(7.3)$ \\
Mean weeks of sick leave (SD) & $9.4(8.2)$ & $8.8(5.0)$ & $9.1(6.7)$ \\
Mean work resumption \% & $16.9(25.9)$ & $14.1(21.9)$ & $15.4(23.9)$ \\
\hline
\end{tabular}

Note. $\mathrm{W}-\mathrm{CBT}=$ work-focused cognitive behavioral therapy; $\mathrm{CBT}=$ cognitive behavioral therapy; $\mathrm{MHC}=$ mental health centre; $S D=$ standard deviation.

* significant difference between the intervention groups at the $p<.05$ level.

assigned to one of the departments based on proximity to their home address. Exceptions were sporadically made in cases of long waiting lists at a certain department (clients were then invited to visit another department). There were no a priori reasons to believe that the clients in the various departments differed with respect to sociodemographic background, severity of complaints, or any other variable.

\section{Interventions}

Cognitive Behavioral Therapy (CBT) was performed according to a protocol that is widely used and acknowledged as state-ofthe-art treatment for work-related mental health problems in the Netherlands (Keijsers et al., 2000). Based on the diagnosed disorder, the therapist could choose out of different versions of this protocol (specific versions exist, e.g., for burnout, adjustment disorder, and depression). Each version of this CBT protocol consists of a basic module that focuses on identification of the problem and on reduction of symptoms (e.g., enhancing mood and activation). After this disorder-specific basic module (covering about six sessions), one or more optional modules were chosen in dialogue with the client for the remaining sessions. It is possible that regular CBT incorporated work issues when clients decided to address this topic. The protocol consists generally of 12 sessions in total (in practice it was 11.4 sessions over the course of 5.7 months, see Table 1).

Work-focused cognitive-behavioral therapy (W-CBT) consisted of the regular treatment (CBT) plus a module focusing on work and the return to work. The work-focused module was integrated in each session according to a newly developed protocol (van Schie, Blonk, \& Lagerveld, 2005), consisting of elements used in similar interventions that were evaluated in earlier trials (Blonk et al., 2006; van der Klink et al., 2003). As described before, it was central to the treatment that therapists addressed work issues in an early phase and used work (and the workplace) as a mechanism or a context to reach their treatment goals (such as activation, time structure, social contact, regular activity, and increasing self-esteem).

To integrate this central idea into each session, the W-CBT treatment consisted first of specific work-related (homework) exercises/interventions that were additional to regular CBT interventions (such as drawing a RTW plan). Second, regular CBT interventions or exercises were framed as much as possible in the work context (such as work-focused psycho-education or work-focused behavioral experiments to challenge dysfunctional thoughts). In addition to these two work-related components, treatment time could also be spent on nonwork issues (e.g., marital problems). However, even in these cases, therapists were encouraged to relate these nonwork issues (at least partly) to work (e.g., by asking how work could help to decrease extensive worrying about marital problems). A more detailed description of the specific workrelated interventions in each subsequent session is described below.

In the first session, a work-related explanation of and perspective on the symptoms was given. For example, "Work can offer several things, such as structure and self-esteem, that are beneficial to your recovery" or "You won't recover from your symptoms just by sitting at home, it would probably even get worse." In the second session job characteristics were inventoried and a problem 
analysis of the work situation was made (i.e., concrete tasks and their frequency and duration were described. In addition, these tasks were ranked, comparable to an "anxiety hierarchy" used in exposure in vivo techniques). In the third session an elaborate, gradual (stepwise) RTW plan was drafted: Therapist and client would agree per step on (a) the activities or tasks the client would perform, (b) for how many hours each task would be performed, and (c) whether altering of workplace characteristics was necessary.

Well-balanced work demands were explicitly stated (e.g., in terms of working hours and/or task complexity) such that secure success experiences and learning experiences would be enhanced. Some clients started, for example, by "attending the weekly team meeting," while others quickly resumed their former tasks for limited periods of time. The presence of beneficial work characteristics (like structure and social contact) was also taken into account in planning RTW. In the fourth and the following sessions, RTW was evaluated, and clients were stimulated to take the next step and, in addition, to extend their work resumption until full return was achieved. During these sessions relapse prevention with respect to RTW was also discussed. In each session clients were encouraged to discuss their plans with their occupational physician and employer. Similar to regular CBT the W-CBT protocol consists generally of 12 sessions in total (in practice this was 11.1 sessions over the course of 5.5 months, see Table 1).

\section{Treatment Integrity}

In order to safeguard adequate implementation of the W-CBT, psychologists in the experimental condition received a group training course (of 1.5 hours twice) in the protocol; this consisted of a lecture (presenting state-of-the-art knowledge on RTW interventions) and an interactive component (e.g., discussing questions and perceived obstacles or doubts raised by the therapists). In addition, the therapist had a meeting with a clinical psychologist from the research team every six weeks. In these meetings, both positive experiences and difficulties with W-CBT experienced by the therapists were discussed and suggestions were made for applying the protocol to participating clients. The attendance of these meetings was high, partly due to the clear support of the management team for the research project. An indication of the treatment integrity was retrieved from the participants, who were asked about six core items of the protocol in the first follow-up questionnaire: (a) analysis of problems at work, (b) inventory of tasks and their current execution possibilities, (c) discussing strategies for RTW, (d) discussing strategies for handling problems when back at work, (e) making a detailed work-resumption plan, and (f) evaluating RTW progress. A composite scale score was computed that included these six items (scale score ranged from 0-6). The experimental group scored significantly higher than the control group $(4.53$ vs. $2.43, F(1,82)=12.67, p=.00)$, indicating adequate treatment integrity. The proportion of clients from the experimental group that reported the presence of the first five elements varied between $54 \%$ (for items 1 and 4) and 84\% (for item 5). Item 6 was reported by $27 \%$ of the clients, but was only applicable to those who had resumed work.

\section{Measures}

Return to work (RTW) was operationalized in several ways, including two time-dependent variables. First, the duration of full RTW was defined as the length of time in calendar days from the start of the treatment until full return to work within 1 year, as reported by the participants. Full return to work was defined as working the number of hours specified in the labor contract, except if this was still on a "therapeutic" basis (with adjusted tasks and/or reduced responsibilities). Second, partial RTW was defined as the length of time between the start of treatment and the first partial increase in working hours. Further, the number of RTW steps (changes in hours worked) from start of treatment until full RTW were calculated for each participant. Based on these RTW steps, information on RTW relapses (a decrease in weekly work hours owing to mental health problems) was extracted. Participants may have taken RTW steps (resuming part of their working hours) before the onset of therapy. These steps were not taken into account in the measure of partial RTW, because they fell outside the time of the intervention. Finally, the percentage of work resumption (as compared with contract hours) at baseline as well as work status (proportion of not, partially, or fully at work) at each measurement wave were calculated.

Mental health problems were operationalized as symptoms of stress, depression, anxiety, and burnout. For all measures higher scores reflect higher levels of mental health problems.

Stress was measured using the 7-item subscale "Stress" from the shortened Depression, Anxiety, \& Stress Scale (DASS-21; Lovibond \& Lovibond, 1995; de Beurs, van Dyck, Marquenie, Lange, \& Blonk, 2001). A 4-point severity scale (from $0=$ not applicable to $3=$ very applicable) was used to measure the extent to which stress had been experienced over the previous week. A sample item is "I had difficulty relaxing." The DASS is a measure with a good reliability and validity (de Beurs et al., 2001). Cronbach's alpha in our study was .92. Stress was measured at every measurement wave, except the second (1 month after baseline).

Depression and Anxiety were measured using two subscales of the Symptom Checklist-90 (SCL-90; Arrindel \& Ettema, 2003; Derogatis, 1977). The subscales depression and (generalized) anxiety consist of 16 items and 10 items, respectively. Items referred on a five-point Likert scale $(1=$ not at all to $5=$ extremely $)$ to the extent to which participants were bothered by symptoms of mental ill health during the previous week (e.g., "Thoughts of ending your life" or "Trembling"). The SCL is a validated measure for evaluation of treatment effects and shows good reliability and validity (Arrindel \& Ettema, 2003). Both scales were of excellent internal consistency, with alphas above .90 . SCL scores were gathered by the therapists at baseline and after approximately 3 and 6 months.

Emotional exhaustion was measured using the subscale (five items) of the Dutch version of the Maslach Burnout Inventory (Schaufeli \& van Dierendonck, 2000). A sample item is "I feel burned out from my work". Items were scored on a 7-point Likert scale $(0=$ never to $6=$ always or daily). This measure has been investigated extensively and psychometric properties are adequate to good (Schaufeli \& van Dierendonck, 2000). Cronbach's alpha was .90 in the present study. Emotional exhaustion was measured at every measurement wave, except the second (1 month after baseline). 
We estimated the average costs to the employer of the W-CBT group compared with the CBT group, based on wages paid during the treatment period until full RTW (direct costs). In Western European countries, 1 day of sickness absence costs an employer about 160 Euro in wages paid (AON, 2010). We calculated the difference in working days (not calendar days) until full RTW between treatment conditions based on contract hours (e.g., seven calendar days of sick leave would account for four working days of sick leave, for those with a 32-hr contract). Per intervention group these working days were multiplied by 160 Euro, and divided by the number of participants per group to estimate the average costs per employee in each group.

\section{Statistical Analyses}

Multilevel analysis were used for our nested data on mental health (stress, emotional exhaustion, competence, depression, and anxiety) using the MlwiN software package (Rasbash et al., 2000). Three levels were discriminated: repeated measurements (first level, varying per outcome measure from three to five measurements, resulting in 504 to 840 occasions), individuals (second level, $n=168$ participants), and therapists (third level, $n=38$ therapists). Multilevel analysis has advantages with respect to dealing with missing data due to panel attrition (i.e., individuals who drop out of the study after one or more measurement occasions). Multilevel analysis leads to unbiased estimates when the panel attrition follows a pattern defined as missing-at-random (for more information see Hox, 2010; Little, Schnabel, \& Baumert, 2000). In a stepwise procedure a final model was built for each mental health indicator. First, the appropriateness of a third-level model (i.e., whether variance in mental health over time was explained by therapists) was tested, including both a linear and a quadratic time component. In the second step, it was tested whether the course of mental health over time was best described using a model including a linear or both a linear and a quadratic component. In the third step, the presence of random slopes (i.e., whether individuals differ in the way their complaints change over time) was tested for each outcome measure. In the final step, several covariates were added to the best-fitting model. These covariates were the intervention type and variables that correlated significantly with the presence of missing values, or that showed differences at baseline between the two intervention types. For those models that included random slopes, an interaction variable (Time* Intervention type) was added to investigate whether the intervention type could explain individual differences in the course of mental health complaints over time. Variables in the equation were not centered, because all included variables had interpretable zero values.

The period until full return to work was analyzed using survival analysis (Cox regression). In survival analysis a curve is generated that, in this study, shows how many (what proportion of) individuals remained absent from work over time as a function of the treatment they received. The time lag used in our study was 1 year. To include participants who had not fully resumed work within this period $(n=12)$, an artificial duration was set at 365 days (censored data). Covariates added in this analysis were those variables that correlated significantly with the presence of missing values, or that showed baseline differences between the two intervention types.

\section{Results}

\section{Baseline Differences}

Randomization checks of both groups were performed for 32 variables including demographics, mental health, and working conditions at baseline, and finally therapeutic characteristics over time using ANOVA and chi-square tests (see Table 1). ${ }^{1}$ The results showed only two significant differences. Clients in the control condition spent less time on a waiting list before the start of treatment $(4.4$ weeks vs. $5.9 ; F(1,157)=11.53, p<.01)$ than clients in the experimental condition. In addition, the control condition consisted of fewer married or cohabiting clients $(67 \%$ vs. $\left.86 \% ; \chi^{2}=8.27, p<.01\right)$. Hence, the analyses focusing on RTW and mental health were corrected for these two variables (i.e., time on waiting list and marital status).

\section{Nonresponse and Drop-Out Analysis}

For a number of the 42 clients that refused to participate, gender $(n=40)$, age $(n=20)$, and/or diagnosis $(n=21)$ were registered. Analyses showed no significant differences on these variables between the respondents and nonrespondents.

Differences between participants without missing questionnaires $(48 \%)$ and participants who failed to return one or more questionnaires $(52 \%)$ were investigated on demographics, mental health condition, therapeutic characteristics, duration of sickness absence at baseline, number of weeks on the waiting list, percentage of work resumption at baseline, and treatment condition. Participants who failed to return one or more questionnaires were longer on the waiting list of the mental health center, $F(1,164)=$ $9.47, p<.01$ and were more often male ( $46 \%$ vs. $34 \%, \chi^{2}=5.0$, $p<.05$ ). Missing data analysis per measure or scale revealed no noteworthy differences from the aggregated analysis per missing questionnaire (measurement wave). Missing data analysis on the information gathered from the mental health center files (SCL depression and anxiety scores) did not yield any differences on any of the above-mentioned variables at the 6-month follow-up.

\section{Return to Work Outcomes}

In Table 2 the return-to-work characteristics at the 1-year follow-up are presented per intervention group. As can be seen, $96 \%$ of the work-focused CBT-group (W-CBT) and $91 \%$ of the regular CBT group had fully resumed work within 1 year. This difference in work status was not significant, $F(1,119)=0.5, p>$ .05. Of the participants who had not fully returned to work, almost all had partially resumed work (99\%). The proportion of participants who had fully resumed work at three $\left(\chi^{2}=5.6, p=<.05\right)$ and 6 months $\left(\chi^{2}=3.95, p=<.05\right)$ after baseline did differ between the groups in favor of the W-CBT group. This suggests that the final step toward full RTW occurred earlier in the W-CBT

\footnotetext{
${ }^{1}$ For reasons of economy, not all variables that were checked are presented in Table 1. The complete variable list and statistical results are available on request from the first author.
} 
Table 2

Return To Work (RTW) Characteristics Per Intervention Group at Follow-Up

\begin{tabular}{|c|c|c|c|c|c|c|c|}
\hline Return to Work Measure & Intervention & $n$ & Mean & & $95 \% \mathrm{CI}$ & $S D$ & Median \\
\hline \multirow[t]{2}{*}{ Proportion full RTW within 3 months } & CBT group & 75 & $21 \%$ & \multirow[t]{2}{*}{$*$} & & & \\
\hline & W-CBT group & 78 & $36 \%$ & & & & \\
\hline \multirow[t]{2}{*}{ Proportion full RTW within 6 months } & CBT group & 75 & $55 \%$ & \multirow[t]{2}{*}{$*$} & & & \\
\hline & W-CBT group & 78 & $73 \%$ & & & & \\
\hline \multirow[t]{2}{*}{ Proportion full RTW within 12 months } & CBT group & 75 & $91 \%$ & & & & \\
\hline & $\mathrm{W}-\mathrm{CBT}$ group & 80 & $96 \%$ & & & & \\
\hline \multirow[t]{2}{*}{ Number of steps until full RTW } & CBT group & 70 & 2.94 & \multirow[t]{2}{*}{ * } & $2.58-3.31$ & 1.53 & 3.0 \\
\hline & W-CBT group & 79 & 4.26 & & $3.75-4.78$ & 2.29 & 4.0 \\
\hline \multirow[t]{2}{*}{ Days until partial $\mathrm{RTW}^{3}$} & CBT group & 68 & 59.46 & \multirow[t]{2}{*}{$*$} & $43.8-75.0$ & 64.34 & 38.0 \\
\hline & $\mathrm{W}-\mathrm{CBT}$ group & 81 & 38.06 & & $28.1-48.0$ & 45.03 & 26.0 \\
\hline \multirow[t]{2}{*}{ Days until full RTW from start treatment } & CBT group & 72 & 175.18 & \multirow[t]{2}{*}{ * } & $149.5-200.8$ & 109.14 & 165.0 \\
\hline & $\mathrm{W}-\mathrm{CBT}$ group & 77 & 136.55 & & $115.4-157.7$ & 93.34 & 100.0 \\
\hline
\end{tabular}

Note. $\quad \mathrm{W}-\mathrm{CBT}=$ work-focused cognitive behavioral therapy; $\mathrm{CBT}=$ regular cognitive behavioral therapy.

* Significant difference between the intervention groups at the $p<.05$ level.

group. This observation is supported by the results on the duration until RTW. With respect to the duration until full and partial RTW, we found a significant difference in favor of the W-CBT group in both the univariate analysis (presented in Table 2) and the Cox regression analysis. Participants receiving W-CBT had a greater chance of full $(H R=1.56, p<.05, S E=.19)$ and partial RTW $(H R=1.59, p<.05, S E=.20)$, indicating a shorter duration until both full and partial RTW in the W-CBT group ${ }^{2}$ (see Figures $2 \&$ 3). Based on the median scores presented in Table 2, it can be seen that full return occurred 65 calendar days earlier and partial return 12 calendar days earlier in the W-CBT group compared with the CBT group.

\section{Return-to-Work Process}

Most participants resumed work gradually: only $7 \%$ went directly from $100 \%$ sick leave to full return to work. About $35 \%$ took two or three steps (each step representing an increase in working hours) until full RTW, and another $35 \%$ needed four to seven steps. The remaining participants resumed work in eight to 11 steps (4\%), did not reach full RTW (6\%), or did reach full RTW but did not provide adequate information on the number of steps (12\%). Inspection of Table 2 shows that participants in the W-CBT group used significantly more steps to full RTW (2.94 vs. 4.26; $F(1,147)=16.72, p<.01)$, indicating a more gradual RTW process.

In 20 cases these RTW steps did not show a simple increasing trajectory over the course of 1 year, but showed relapses ("wavelike" fluctuations). Only four cases experienced a relapse after an occurrence of full RTW. These cases were all fully returned again at the 1-year follow-up. Although participants receiving W-CBT experienced a RTW relapse more often $(15.7 \%$ in the W-CBT group vs. $7.6 \%$ in the CBT group), this difference was not statistically significant $\left(\chi^{2}=2.64, p=.10\right)$.

\section{Effects on Mental Health Problems}

Means and standard deviations of mental health complaints for both intervention groups are presented in Table 3, including follow-up scores at 1, 3, 6, and 12 months. At 12 months $98 \%$ of the total participant group had finished treatment; at 6 months (the average treatment duration) this was $65 \%$. Although all mental health problems revealed a decrease at each subsequent measurement wave, average levels of complaints at the 6- and 12-month follow-up remained elevated compared with healthy populations (Arrindel \& Ettema, 2003; Henry \& Crawford, 2005; Schaufeli \& van Dierendonck, 2000). In Table 4 the proportion of participants is presented that had recovered from their mental health problems at that measurement wave. At the 1-year follow-up almost $70 \%$ had recovered from burnout and around $50 \%$ had recovered from stress. At the 6-month follow-up (the average treatment duration) more than half of the sample was recovered from both anxiety and depression. The proportions of recovered employees did not differ between the treatment types for any of the mental health indicators.

To examine the effects of the W-CBT on the course of mental health problems, multilevel analysis was conducted. For "Stress" and "Emotional Exhaustion," five measurement waves were analyzed over a period of 1 year. For depression and anxiety three measurement waves were used over a period of 6 months.

For none of the mental health indicators could a three-level structure be found within the data. This indicates that differences in mental health problems over time were not explained by the differences between individual therapists. A negative linear time component was observed in each outcome variable ( $p<.05, z$ values varied between 4.87 and 10.5), indicating that all mental health problems decreased over time. A (positive) quadratic component was present for emotional exhaustion, stress, and depression $(p<.05, z$ values varied between 3.0 and 7.3), but not for anxiety $(z=1.24, p=.21)$. These quadratic components showed that the decrease in complaints was steeper in the first months for exhaustion, stress, and depression. A comparison with the fixed model revealed the presence of random slopes in the equations for emotional exhaustion $\left(\chi_{(1)}^{2}=6.2, p<.05\right)$ and stress $\left(\chi_{(1)}^{2}=12.5, p<.01\right)$, but not for depression and anxiety. Thus, clients varied significantly in how their levels of exhaustion and

\footnotetext{
${ }^{2}$ Both the analyses on full and partial RTW were corrected for baseline differences and missingness.

${ }^{3}$ Those variables that were identified as relevant to RTW trajectories of our target population were included (Cornelius, van der Klink, Groothoff, \& Brouwer, 2011; Lagerveld et al., 2010b).
} 


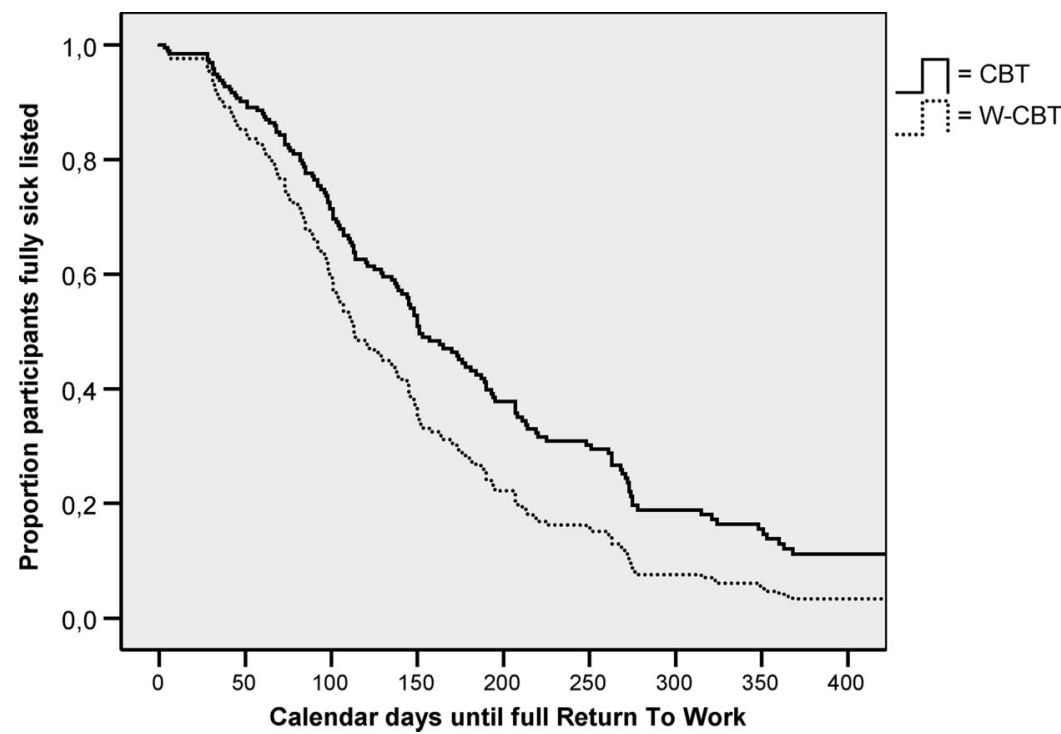

Figure 2. Cumulative probability (survival function) of being fully sick-listed from the start of treatment in the "cognitive behavioral therapy" group (CBT) and the "work-focused CBT" group (W-CBT).

stress changed over time. However, this individual variation could not be explained by the treatment condition as none of the Time* Condition interaction terms (for exhaustion and stress) in the final model were significant. Similar to what was seen in the earlier models, the final model presented in Table 5 shows that a linear decrease over time was present for all mental health indicators, and this decrease was steeper in the first months (positive quadratic component) for exhaustion, depression, and stress.

\section{Economic Consequences for the Employer}

The shorter duration until full RTW in the W-CBT group has financial advantages for employers who pay wages to absent and nonproductive employees. Calculations showed that employers in the CBT group paid on average $€ 16,727$ (US $\$ 24,220$ ) in wages per employee during entire the sick leave period from the start of the intervention until full RTW, whereas employers in the W-CBT group paid $€ 13,085$ (US $\$ 18,952$ ) ( $€ 3,642$ or US $\$ 5,275$ difference). This implies a $20 \%$ cost reduction for employers whose employees receive W-CBT.

\section{Discussion}

To our knowledge, this was the first study in which workfocused cognitive-behavioral therapy (CBT) carried out by psy-

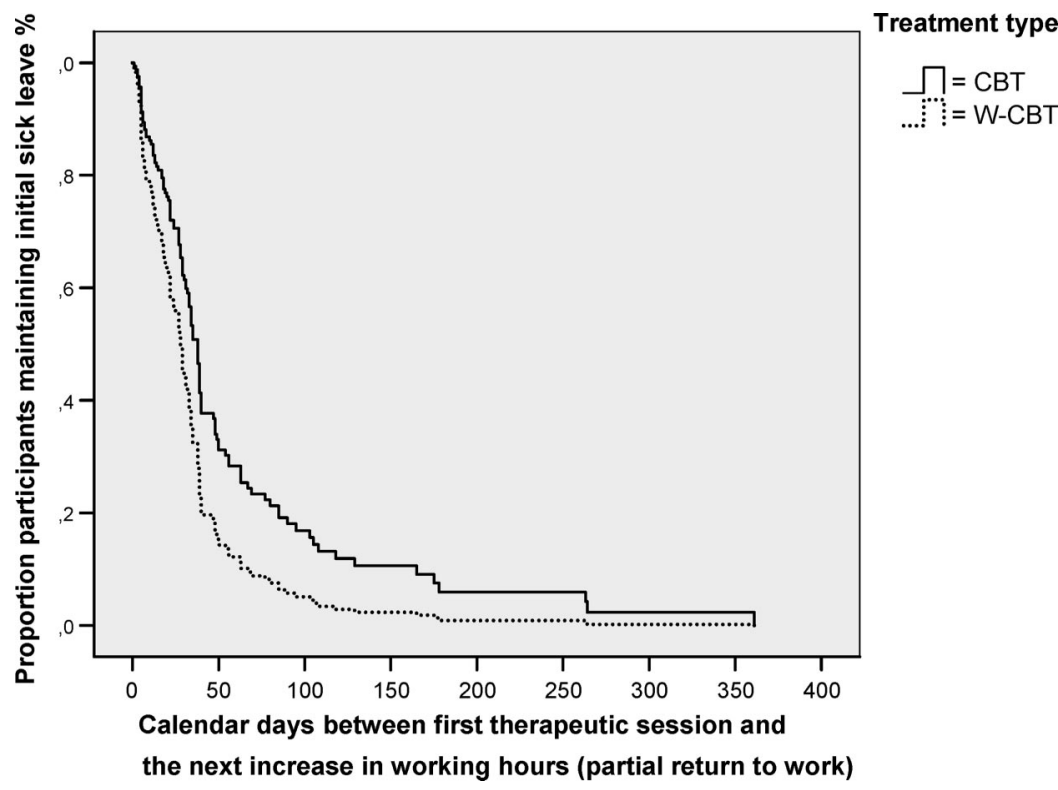

Figure 3. Cumulative probability (survival function) of maintaining the initial sick leave level from the start of treatment in the "cognitive behavioral therapy" group (CBT) and the "work-focused CBT" group (W-CBT). 
Table 3

Means (and Standard Deviations) of Mental Health Problems Per Intervention Group

\begin{tabular}{|c|c|c|c|c|c|c|c|c|c|}
\hline Mental Health Problem & Group & Baseline & $n$ & 3-month follow-up & $n$ & 6-month follow-up & $n$ & 12-month follow-up & $n$ \\
\hline \multirow[t]{2}{*}{ Stress } & CBT & $12.8(4.9)$ & 78 & $8.3(5.7)$ & 58 & $6.49(4.5)$ & 53 & $5.5(4.7)$ & 49 \\
\hline & W-CBT & $11.4(4.7)$ & 89 & $7.1(4.6)$ & 60 & $6.42(4.3)$ & 55 & $6.3(5.2)$ & 49 \\
\hline \multirow[t]{2}{*}{ Depression } & CBT & $40.1(11.8)$ & 78 & $29.1(11.9)$ & 68 & $21.8(7.5)$ & 32 & - & \\
\hline & W-CBT & $38.9(10.4)$ & 86 & $26.8(9.1)$ & 70 & $22.8(8.3)$ & 32 & - & \\
\hline \multirow[t]{2}{*}{ Anxiety } & CBT & $21.9(8.3)$ & 78 & $17.6(7.8)$ & 68 & $13.4(4.9)$ & 32 & - & \\
\hline & W-CBT & $22.5(8.3)$ & 86 & $17.0(5.8)$ & 70 & $13.6(4.7)$ & 32 & - & \\
\hline \multirow[t]{2}{*}{ Emotional Exhaustion\# } & $\mathrm{CBT}$ & 3.5 (1.7) & 79 & $2.5(1.5)$ & 49 & $2.16(1.2)$ & 48 & $1.65(1.2)$ & 46 \\
\hline & W-CBT & $3.3(1.8)$ & 89 & $2.4(1.3)$ & 54 & $2.41(1.5)$ & 51 & $1.97(1.5)$ & 45 \\
\hline
\end{tabular}

Note. $\quad \mathrm{W}-\mathrm{CBT}=$ work-focused cognitive behavioral therapy; CBT $=$ cognitive behavioral therapy.

\# Because of its work relatedness, emotional exhaustion was only measured at follow-up when participants had (partially) resumed their work.

chotherapists was compared with regular CBT, among employees on sick leave due to common mental disorders. In line with our expectations we found that clients who received the work-focused treatment (W-CBT) resumed work earlier than those who received regular CBT. Psychological complaints declined significantly over time. As expected, no difference in complaint reduction was observed between the treatment groups.

\section{Return to Work: Main Findings and Interpretations}

Concerning our main outcome measure, return to work, we found robust and large effects in favor of W-CBT: these clients fully resumed work 65 days earlier than clients receiving regular CBT. Most clients (over 90\%) from both groups had resumed work within 1 year, but W-CBT achieved this result about 2 months earlier. We also found that gradual work resumption occurred earlier and was implemented more often in the W-CBT intervention group. Participants in the W-CBT group reported earlier partial RTW and used more (and consequently smaller) steps to reach full RTW compared with those in the regular CBT group. Finally, temporal relapses in the RTW process occurred more often in W-CBT, but this difference was not statistically significant. These results largely support the first hypothesis (1a).

Outcome studies in similar target groups show a large range in reported RTW outcomes. The current findings are within the upper range compared with these other studies, for both "time until full RTW" and "levels of RTW" at 1-year follow-up (de Vente et al., 2008; Nieuwenhuijsen, 2004; van der Klink et al., 2003, and see for a review Rebergen, 2009). In addition, the effectiveness of
W-CBT in promoting RTW is in line with study findings that suggest that CBT as currently practiced, without a specific focus on work (resumption), has little effect on RTW (Blonk et al., 2006; de Vente et al., 2008).

Based on theories underlying CBT (e.g., Bandura, 1977; Beck, 1976; Meichenbaum \& Cameron, 1982), the effectiveness of W-CBT might be explained by various mechanisms, both from a cognitive perspective (e.g., dysfunctional work-related beliefs have changed) and a behavioral view (e.g., RTW related behavior was reinforced). Our results suggest that work-directed gradual exposure (that is well guided early and partial work resumption) is an important factor in explaining the effectiveness of W-CBT. The shorter time until full RTW in the W-CBT group may (in part) be attributed to earlier partial RTW: "well begun is half done." This notion needs to be investigated more extensively, but is supported by preliminary findings in an earlier study (Blonk \& Lagerveld, 2007). In addition, our observations during the meetings with the therapists support the idea that W-CBT is predominately distinguished from regular CBT by the focus on early and gradual RTW. Stimulating both early partial RTW and the final step toward full RTW by creatively integrating these techniques into regular CBT interventions were important issues that required the therapists to change their regular ways of thinking and acting. The findings of one other study in which CBT offered by clinical psychologists was evaluated may shed additional light on the importance of stimulating early and gradual RTW (de Vente, Kamphuis, \& Emmelkamp, 2001). This CBT intervention addressed work issues, thereby predominantly focusing on mental (and physical) health

Table 4

Proportion of Clients Recovered From Mental Health Problems at Each Measurement Wave

\begin{tabular}{|c|c|c|c|c|c|c|c|c|c|}
\hline & Group & baseline & $n$ & 3-month follow-up & $n$ & 6-month follow-up & $n$ & 12-month follow-up & $n$ \\
\hline \multirow[t]{2}{*}{ Recovered from burnout (\%) } & CBT & 25.3 & 79 & 36.7 & 49 & 54.2 & 48 & 69.6 & 46 \\
\hline & W-CBT & 36.0 & 89 & 50.0 & 54 & 52.9 & 51 & 66.7 & 45 \\
\hline \multirow[t]{2}{*}{ Recovered from stress (\%) } & $\mathrm{CBT}$ & 5.1 & 78 & 32.8 & 58 & 39.6 & 53 & 53.1 & 49 \\
\hline & W-CBT & 6.7 & 89 & 36.7 & 60 & 36.4 & 55 & 49.0 & 49 \\
\hline \multirow[t]{2}{*}{ Recovered from anxiety $(\%)$} & CBT & 14.1 & 78 & 33.8 & 68 & 59.4 & 32 & - & \\
\hline & W-CBT & 9.3 & 86 & 30.0 & 70 & 53.1 & 32 & - & \\
\hline \multirow[t]{2}{*}{ Recovered from depression (\%) } & CBT & 2.6 & 78 & 36.8 & 68 & 65.6 & 32 & - & \\
\hline & W-CBT & 1.2 & 86 & 35.7 & 70 & 62.5 & 32 & - & \\
\hline
\end{tabular}

Note. $\quad$ W-CBT $=$ work-focused cognitive behavioral therapy; CBT = cognitive behavioral therapy. Thresholds used: 2.20 (burnout), 4.72 (stress), 12 (anxiety males), 14 (anxiety females), 21 (depression males), 23 (depression females). 
Table 5

Results Multilevel Analysis: Effect of Intervention Type on the Course of Anxiety, Depression, Stress, and Emotional Exhaustion Over Time

\begin{tabular}{|c|c|c|c|c|c|c|c|c|}
\hline \multirow[b]{2}{*}{ Parameter } & \multicolumn{2}{|c|}{ Exhaustion\# } & \multicolumn{2}{|c|}{ Depression } & \multicolumn{2}{|c|}{ Anxiety } & \multicolumn{2}{|c|}{ Stress } \\
\hline & $B$ & $S E$ & $B$ & $S E$ & $B$ & $S E$ & $B$ & $S E$ \\
\hline Intercept & 3.46 & .29 & 42.96 & 2.05 & 23.39 & 1.44 & 13.39 & .89 \\
\hline Time & $-.24^{*}$ & .04 & $-4.62^{*}$ & .59 & $-1.48^{*}$ & .14 & $-1.33^{*}$ & .13 \\
\hline Time $^{2}$ & $.01^{*}$ & .00 & $.29^{*}$ & .11 & - & - & $.07 *$ & .01 \\
\hline Condition $(1=\mathrm{W}-\mathrm{CBT})$ & -.22 & .23 & -.64 & 1.51 & .29 & 1.07 & -.91 & .71 \\
\hline Condition * time & .03 & .03 & - & - & - & - & .12 & .08 \\
\hline Loglikelihood (deviance) & \multicolumn{2}{|c|}{1755.334} & \multicolumn{2}{|c|}{2484.080} & \multicolumn{2}{|c|}{2235.535} & \multicolumn{2}{|c|}{3057.498} \\
\hline Time coding (months) & \multirow{2}{*}{\multicolumn{2}{|c|}{$0,3,6,9,12$}} & \multicolumn{2}{|c|}{$0,3,6$} & \multicolumn{2}{|c|}{$0,3,6$} & \multicolumn{2}{|c|}{$0,3,6,9,12$} \\
\hline $\mathrm{N}$ observations & & 507 & \multicolumn{2}{|c|}{338} & \multicolumn{2}{|c|}{338} & \multicolumn{2}{|c|}{536} \\
\hline
\end{tabular}

Note. $\quad$ W-CBT $=$ work-focused cognitive behavioral therapy; Time squared was not included in the final model when the first model did not reveal a significant effect of a time-squared component; The Condition * Time interaction term was not included in models without random slopes; All analysis were corrected for baseline differences between the intervention groups and selective missingness (gender, partner, and weeks on the waiting list).

* Significant difference between the intervention groups at the $p<.05$ level. \# Because of its work relatedness, emotional exhaustion was only measured at follow-up when participants had (partially) resumed their work.

and following a symptom-contingent approach. This CBT intervention appeared not to be effective in promoting RTW, though. The more favorable outcomes of our alternative W-CBT intervention may be explained by an earlier, more integrated focus on RTW, including full RTW. As mentioned in the introduction, we expected that gradual RTW might be a mechanism that promotes full RTW because it enables people to acquire the necessary coping skills to deal with (return to) work stressors and practice these skills in the workplace. In line with the CBT technique of gradual exposure and graded activity, partial work resumption may challenge dysfunctional cognitions and secure success experiences at work. An important cognition that could explain the effectiveness of W-CBT may be "work-related self-efficacy," that is, the belief that one can fulfill one's work demands or work role (Lagerveld, Blonk, Brenninkmeijer, \& Schaufeli, 2010a). When the RTW process is adequately guided, resulting in mastery experiences, self-efficacy theory would predict that employees will be more persistent and successful in their RTW process (Bandura, 1977; Bandura, 1986; Lagerveld et al., 2010a). Possible effective components in W-CBT such as early RTW, gradual RTW and the promotion of work-related self-efficacy should be further clarified in future research.

An obvious concern is that (early) work resumption might increase relapses in sick leave, when workers have not fully recovered from their symptoms. In the process toward full work resumption, W-CBT participants experienced somewhat more relapses (although this was not a statistically significant difference). These relapses were, however, not of a permanent nature, but showed a wave-like pattern leading to full RTW within 1 year. This may indicate that participants in the W-CBT use more experimental learning with respect to RTW. RTW relapses had no adverse effect on mental health problems over the course of 1 year. Beneficial effects of early gradual RTW should, however, be interpreted within the context of the treatment offered. The gradual RTW process in W-CBT was well guided and included many creative, tailored RTW strategies (not only gradually increasing work hours)
To conclude, we argue that employees receiving CBT with an integrated, tailored and early focus on (gradual return to) work resume their work faster than employees receiving regular CBT.

\section{Economic Consequences}

The outcomes on RTW may have important consequences for employers as well. The cost reduction for an employer in the Netherlands was estimated at 20\% (US $\$ 5,275$ gain for an average individual sick leave case) per employee, based only on wages paid during sickness absence. As the work-focused treatment did not entail additional costs (e.g., the interventions did not differ with respect to contact hours or number of professionals involved), one might assume this intervention to be economically superior to regular care. The current estimations support Hypothesis 1b. Although the absolute savings for a single case are moderate, a $20 \%$ reduction of costs associated with sick leave due to mental health problems is of relevance, considering the prevalence numbers of this type of absenteeism (e.g., mental disorders account for $30 \%$ of long-term sick leave in the Netherlands, UWV, 2007). Finally, the estimations were rather conservative, as we did not incorporate any other associated costs, such as productivity loss and hiring of replacement costs. To draw firmer conclusions about cost effectiveness, net cost-benefit analysis should be performed in future research. These analyses should take the above mentioned additional costs into account, as well as incorporate (more detailed) information on other direct and indirect costs (e.g., employers' insurance against sick leave payments, costs of the intervention, other health care utilization costs, and wages on the individual level; AON, 2010; Joensuu \& Lindström, 2003; Rebergen, 2009).

\section{Mental Health Problems: Main Findings and Interpretations}

In line with our expectations (Hypothesis 2), we found that all mental health problems decreased over time, irrespective of the type of treatment. In addition, both interventions achieved (on average) clinical recovery for emotional exhaustion in 12 months 
and near recovery levels for depression and anxiety at 6 months. Stress levels, however, remained clearly elevated compared with healthy controls.

These results are in line with those of other studies that reported elevated symptom levels at follow-up and equal patterns of symptom reduction for CBT-based RTW interventions compared with care as usual (Blonk et al., 2006; de Vente et al., 2008; Nieuwenhuijsen, 2004; Schene et al., 2007; van der Klink et al., 2003).

Several explanations may be proposed for our finding that W-CBT was more effective with respect to RTW, but not for the reduction of symptoms. First, a temporal increase of symptoms evoked by confrontation with stressors while returning to work might be counterbalanced by the beneficial effects of work at the same time, or later. In the current study, time-spans were used that appear to be too long to unravel these patterns. Second, the current results on RTW and mental health complaints can be seen as an indication that psychotherapists in the W-CBT group managed to implement a less symptom-contingent (but more time-contingent) approach. Clients with similar symptom levels were more often successfully motivated to RTW in the W-CBT group compared with the regular CBT group. In the latter group psychologists may have waited until symptoms were further reduced. Finally, it may be assumed that CBT (offered to both groups) already leads to the best achievable results, and that the beneficial influences of work can hardly contribute to a further reduction of complaints. We have to note, however, that the effectiveness of regular CBT in reducing the symptoms of employees with common mental health problems is a subject of debate (de Vente et al., 2008; Blonk et al., 2006). As we did not compare the W-CBT treatment with a no-treatment control group, we cannot test this critique. However, even if the observed symptom reduction in this study was caused by natural recovery, it can be expected that this process would occur in both groups equally.

The elevated levels of mental health problems at follow-up may be a point of concern with respect to RTW, as even subthreshold levels have been related to increased work-loss days in the future (Rai, Skapinakis, Wiles, Lewis, \& Araya, 2010). These results can be explained by the time of measurement (at the depression and anxiety follow-up only $65 \%$ of the participants had finished their treatment) or by higher original stress levels, as suggested in earlier studies (Blonk et al., 2006; de Vente et al., 2008). Perhaps the participants in our sample had regained their initial levels of stress, which were higher compared with a healthy reference group.

We conclude that W-CBT does not impede recovery from psychological complaints, compared with regular therapy.

\section{Limitations}

An important limitation of our study is the lack of patient randomization: such a randomized design is considered the stateof-the-art design for intervention studies. The consequential potential biases should be kept in mind when interpreting our findings (i.e., attributing the effects to differences in treatment). However, several points may contribute to the robustness and validity of our findings. First, the allocation of participants to the intervention occurred centrally for all departments. This process did not involve content-driven choices on the part of either the therapist or the client. Second, the analysis performed on baseline group differences showed two significant differences out of the 32 variables investigated. ${ }^{3}$ These results could, however, be statistically expected based on the number of analyses performed at the .05 level. Controlling for these two significant variables in our statistical analysis did not yield any noteworthy differences. In addition, we conducted some exploratory analyses to examine the influence of potential other relevant variables (e.g., educational level, age, work characteristics, severity of mental health complaints) on "time to RTW." The results indicated the robustness of the effect of W-CBT. Although we cannot completely rule out the theoretical influence of "unobserved third-variables," we believe that it is not very likely that other variables have played a crucial role in the RTW outcomes.

A second limitation concerns the restrictions of our mental health data. First, for all mental health outcomes, substantial dropout occurred; however, this was not selective for most of the variables studied. Even though we controlled for the few variables that revealed selective dropout, our results might be biased. Furthermore, we were unable to gather information on psychological well-being in the longer-term and at critical RTW events (e.g., during increases in work hours). Our follow-up period did not exceed 1 year, although it is not uncommon for clients to experience relapses after this, especially among those suffering from depression (Westen, Novotny, \& Thompson-Brenner, 2004). Therefore, we cannot draw any conclusions about the long-term effectiveness of the treatments. This is especially the case with respect to depression and anxiety, as we were able to collect information on these mental health indicators only at the 6-month follow-up.

Another point of concern is the limited amount of information about treatment integrity. Data obtained from the clients indicate, nevertheless, that work elements were more often present in the W-CBT treatment condition. Moreover, the results on partial RTW and the RTW process also support the treatment integrity: As described in the protocol, therapists in the W-CBT group managed to stimulate gradual and early work resumption during the therapeutic sessions by integrating work-related aspects early into therapy. Finally, because of the separate team structures, there was no spillover of W-CBT knowledge or strategies from the therapists of $\mathrm{W}-\mathrm{CBT}$ group to those of the CBT group.

\section{Strengths: Contribution to Knowledge on Effective RTW Interventions}

To our knowledge, this was the first study in which an integrated work-focused CBT treatment carried out by psychotherapists was compared with regular CBT. Our findings may, therefore, offer a valuable contribution to the current knowledge of RTW interventions for workers with common mental health problems. As described in the introduction, controlled intervention studies in this field are rare, especially for interventions provided by mental health professionals. This can be partly attributed to the fact that not many researchers succeed in establishing a randomized trial, owing to ethical and practical constraints (Lander et al., 2009;

\footnotetext{
${ }^{4}$ As this variable was not normally distributed (skewness: 2.8, kurtosis: 9.8) the nonparametric Mann Whitney test was used to compare the groups.
} 
Myette, 2008; Rebergen, 2009). There is, however, a great need for more evidence-based action within the field of reintegration, especially for clients with mental health problems. We hope that this quasi-experimental study can serve as a fruitful basis for designing future, more rigorously controlled RCT's.

Because two very similar treatments were compared in this study, it was possible to gain a better impression of the additional value of a work-related focus. Even within RCT designs, the comparison of interventions applied by different professionals or at different levels of intensity often leads to difficulty in explaining intervention effects. It is also important to note that our CBT condition can truly be viewed as "care as usual," as this type of treatment is the "treatment of choice" for many different disorders worldwide (Butler et al., 2006). Furthermore, as our sample consisted of a substantial number of employees, with a variety of common mental health complaints (including minor depression), and with a variety of jobs, our study may have good external generalizability for the population of employees with (common) mental health disorders.

The results of our study are unique in the sense that they show the promising effects of a work-focused intervention that can be integrated easily into regular psychological therapy at relatively low costs. Contrary to the findings of earlier studies (Bakker et al., 2007; Brouwers, Tiemens, Terluin, \& Verhaak, 2006), the current findings show that using W-CBT, professionals with a rather distal relation to the workplace, such as psychotherapists, are able to enhance their effectiveness in promoting RTW.

\section{Other Recommendations for Future Research}

First, our results should be replicated in a randomized controlled design. Ideally, a future RCT should include a less intensive treatment condition (such as CBT-based treatment provided by less expensive professionals) next to a treatment-as-usual condition (regular CBT). Such a three-armed trial could address the question whether making psychotherapy more work-focused is the most (cost) effective solution.

Second, future research should also include workers with more severe disorders, like major depression, which is expected to become one of the leading causes of work disability (WHO, 2002).

Finally, future research could improve the definition and operationalization of the complex concept of RTW (Wasiak et al., 2007): for example, by paying more attention to its sustainability (e.g., relapses), the quality of RTW (e.g., work functioning), and the views of different stakeholders on successful RTW. As variations in the operationalization of RTW can lead to different conclusions about treatment effectiveness, it is important to use a variety of indicators to measure RTW in future studies.

\section{Practical Implications}

Based on the promising results of this quasi-experimental study, mental health professionals are recommended to discover how a more profound focus on early and gradual RTW, using W-CBT, works in their practice. A successful implementation of W-CBT may depend on several factors, such as the attitudes and skills of clinicians, and RTW policies.

The fact that CBT is the worldwide treatment of choice for many common mental disorders provides many opportunities for implementation. Clinicians currently providing CBT may be stimulated to apply W-CBT when they are reassured by our mental health outcomes in the still-common fear that symptoms will increase with early RTW (Kidd et al., 2008; van Oostrom, et al., 2010). Furthermore, it was our experience that the therapists from the W-CBT group were able to change protective, symptomcontingent habits because of the project meetings and positive experiences when implementing the protocol in practice.

In addition, we feel that a fundamental debate within the mental health service sector on the role of work issues is needed. Longterm sick leave due to mental ill health is a multifactorial problem that requires stakeholder collaboration and the attuning of different treatments to one another (Vlasveld et al., 2009). Mental health professionals might help break the boundaries by integrating knowledge from occupational professionals with their own expertise on the treatment of mental health problems.

It is important to note that, to obtain the most favorable effects from W-CBT, RTW policies that allow individuals to return to work, fully or gradually, should be present. These RTW policies will be dependent on national social security systems (Stress Impact, 2006). For instance, offering modified work to (temporarily) disabled workers is possible in several countries such as the United States, Canada, Australia, the Netherlands, and the U.K. (Krause, Dasinger, \& Neuhauser, 1998). We expect that in the near future more and more countries will develop policies to facilitate (gradual) RTW (Rebergen, 2009), which will increase the practical relevance and cross-cultural generalizability of our findings.

\section{Conclusion}

In sum, our findings showed that through focusing more and earlier on work-related aspects, psychotherapists can substantially speed up RTW in employees. This result can be achieved without negative side effects on symptom recovery. Integrating workrelated aspects with cognitive-behavioral therapy can be a fruitful approach with benefits for employees, employers, and care providers.

\section{References}

Andrea, H., Bultmann, U., Beurskens, A. J., Swaen, G. M., van Schayck, C. P., \& Kant, I. J. (2004). Anxiety and depression in the working population using the HAD Scale, psychometrics, prevalence and relationships with psychosocial work characteristics. Social Psychiatry and Psychiatric Epidemiology, 39, 637-646.

Anema, J. R., Steenstra, I. A., Bongers, P. M., de Vet, H. C. W., Knol, D. L., \& van Mechelen, W. (2007). Multidisciplinary rehabilitation for subacute low back pain: Graded activity or workplace intervention or both? A randomized controlled trial. Spine, 32, 291-298. doi:10.1097/ 01.brs.0000253604.90039.ad

AON Consultancy. (2010). The European Sick Leave Index. Fresh Insights into Sick Leave Absence. This document was retrieved from www .aon.com/Netherlands/campagnes/ESLI/ESLI.jsp., on November 2, 2010.

APA. (1994). Diagnostic and Statistical Manual of Mental Disorders (DSM-IV) 4th Edition. Washington, DC: American Psychiatric Association.

Arnetz, B. B., Sjögren, B., Rydéhn, B., \& Meisel, R. (2003). Early workplace intervention for employees with musculoskeletal-related absenteeism: A prospective controlled intervention study. Journal of Oc- 
cupational and Environmental Medicine, 45, 499-506. doi:10.1097/ 01.jom.0000063628.37065.45

Arrindel, W. A., \& Ettema, J. H. M. (2003). Symptom Checklist SCL-90: Handleiding bij een multidimensionele psychopathologie-indicator [Manual to a multidimensional psychopathology-indicator]. Lisse, The Netherlands: Swets Test Publishers.

Bakker, I. M., Terluin, B., van Marwijk, H. W., van der Windt, D. A., Rijmen, F., van Mechelen, W., \& Stalman, W. A. (2007). A clusterrandomised trial evaluating an intervention for patients with stressrelated mental disorders and sick leave in primary care. PLoS Clinical Trials, 2, e26. doi:10.1371/journal.pctr.0020026

Bandura, A. (1977). Self-efficacy: Toward a unifying theory of behavioral change. Psychological Review, 84, 191-215. doi:10.1037/0033295X.84.2.191

Bandura, A. (1986). The explanatory and predictive scope of self-efficacy theory. Journal of Social and Clinical Psychology, 4, 359-373. doi: 10.1521/jscp.1986.4.3.359

Beck, A. T. (1976). Cognitive therapy and the emotional disorders. New York, NY: International Universities Press.

Beck, A. T., Rush, A. J., Shaw, B. F., \& Emery, G. (1979). Cognitive therapy of depression. New York, NY: Guilford Press.

Blonk, R. W. B., Brenninkmeijer, V., Lagerveld, S. E., \& Houtman, I. L. D. (2006). Treatment of work-related psychological complaints: A randomised field experiment among self-employed. Work \& Stress, 20, 129-144. doi:10.1080/02678370600856615

Blonk, R. W. B., \& Lagerveld, S. E. (2007, May). Early partial return to work and its effects on full return to work. Presentation at the EAWOP Congress, Stockholm, Sweden.

Boedeker, W., \& Klindworth, H. (2007). Hearts and minds at work in Europe. A European work-related public health report on cardiovascular diseases and mental ill health. Essen, Germany: BKK Bundesverband.

Bowling, A. (1995). What things are important in people's lives? A survey of the public's judgements to inform scales of health related quality of life. Social Science \& Medicine, 41, 1447-1462. doi:10.1016/02779536(95)00113-L

Brouwers, E. P. M., Tiemens, B. G., Terluin, B., \& Verhaak, P. F. M. (2006). Effectiveness of an intervention to reduce sickness absence in patients with emotional distress or minor mental disorders: A randomised controlled effectiveness trial. General Hospital Psychiatry, 28, 223-229. doi:10.1016/j.genhosppsych.2006.02.005

Butler, A. C., Chapman, J. E., Forman, E. M., \& Beck, A. T. (2006). The empirical status of cognitive-behavioral therapy: A review of metaanalyses. Clinical Psychology Review, 26, 17-31. doi:10.1016/ j.cpr.2005.07.003

Cornelius, L. R., van der Klink, J. J. L., Groothoff, J. W., \& Brouwer, S. (2011). Prognostic factors of long term disability due to mental disorders: A systematic review. Journal of Occupational Rehabilitation, 21, 259-274. doi:10.1007/s10926-010-9261-5

de Beurs, E., van Dyck, R., Marquenie, L. A., Lange, A., \& Blonk, R. W. B. (2001). De DASS: Een vragenlijst voor het meten van depressie, angst en stress. [The DASS: A questionnaire for the measurement of depression, anxiety, and stress]. Gedragstherapie, 34, 35-53.

Derogatis, L. R., (1977). SCL-90: Administration, scoring and procedures manual-I for the revised version. Baltimore, MD: Johns Hopkins University school of Medicine, Clinical Psychometrics Research Unit.

de Vente, W., Kamphuis, J. H., Emmelkamp, P. M. G., \& Blonk, R. W. B. (2008). The effects of cognitive behavioral treatment on work-related stress complaints and sickness absence: A randomized controlled trial. Journal of Occupational Health Psychology, 13, 214-231. doi:10.1037/ 1076-8998.13.3.214

de Vente, W., Kamphuis, J. H., \& Emmelkamp, P. M. G. (2001). Stress Management Training individual protocol, manual. Unpublished manuscript, University of Amsterdam, Department of Clinical Psychology.
Durand, M. J., \& Loisel, P. (2001). Therapeutic return to work: Rehabilitation in the workplace. Work: A Journal of Prevention, Assessment \& Rehabilitation, 17, 57-63.

Ellis, A. (1997). Stress counselling: A rational emotive behaviour approach. London, UK: Cassell.

Glozier. (2002). Mental ill health and fitness for work. Occupational and Environmental Medicine, 59, 714-720.

Goetzel, R. Z, Hawkins, K., Ozminkowski, R. J., \& Wang, S. (2003). The health and productivity cost burden of the "Top 10" physical and mental health conditions affecting six large U.S. employers in 1999. Journal of Occupational and Environmental Medicine, 45, 5-14. doi:10.1097/ 00043764-200301000-00007

Goetzel, R. Z., Ozminkowski, R. J., Sederer, L. I., \& Mark, T. L. (2002). The business case for quality mental health services: Why employers should care about the mental health and well-being of their employees. Journal of Occupational and Environmental Medicine, 44, 320-330. doi:10.1097/00043764-200204000-00012

Greenberg, P. E., Kessler, R. C., Birnbaum, H. G., Leong, S. A., Lowe, S. W., Berglund. P. A., \& Corey-Lisle, P. K. (2003). The economic burden of depression in the United States: How did it change between 1990 and 2000? Journal of Clinical Psychiatry, 64, 1465-1475. doi: 10.4088/JCP.v64n1211

Henry, J. D., \& Crawford, J. R. (2005). The short-form version of the Depression Anxiety Stress Scales (DASS-21): Construct validity and normative data in a large non-clinical sample. British Journal of Clinical Psychology, 44, 227-239. doi:10.1348/014466505X29657

Hobbis, I. C. A., \& Sutton, S. (2005). Are techniques used in cognitive behaviour therapy applicable to behaviour change interventions based on the theory of planned behaviour? Journal of Health Psychology, 10, 7-18. doi:10.1177/1359105305048549

Hox, J. (2010). Multilevel analysis: Techniques and applications. Second edition. New York, NY: Routledge Academic.

Joensuu, M., \& Lindström, K. (2003). Sickness absence and stress factors at work. Finnish Institute of Occupational Health. Stress Impact Report, Chapter 3. Retrieved from www.surrey.ac.uk/Psychology/stressimpact/ publications

Keijsers, G. P. J., Schaap, C. P. D. R., Vossen, C. J. C., Boelaars, V., van Minnen, A., \& Hoogduin, C. A. L. (2000). Burnout reïntegratietraining, therapeutenboek [Burnout reintegration training, therapist book]. Amsterdam, The Netherlands: Boom Cure \& Care Publishers.

Kessler, R. C., Akiskal, H. S., Ames, M., Birnbaum, H. Greenberg, P., Hirschfeld, R., . . . Wang, P. S. (2006). Prevalence and effects of mood disorders on work performance in a nationally representative sample of U.S. workers. American Journal of Psychiatry, 163, 1561-1568. doi: 10.1176/appi.ajp.163.9.1561

Kessler, R. C., Berglund, P., Demler, O., Jin, R., Koretz, D., Merikangas, K. R., . . Wang, P. S. (2003). The epidemiology of major depressive disorder: Results from the National Comorbidity Survey Replication (NCS-R). Journal of the American Medical Association, 289, 30953105. doi:10.1001/jama.289.23.3095

Keuzenkamp, H., Kok, L., \& Seters, J. (2002). Reguliere zorg is niet gericht op reïntegratie [Regular care is not directed towards reintegration] Tijdschrift voor Bedrijfs-en Verzekeringsgeneeskunde, 10, 363 366. doi:10.1007/BF03073811

Kidd, S. A., Boyd, G. M., Bieling, P., Pike, S., \& Kazarian-Keith, D. (2008). Effect of a vocationally-focused brief cognitive behavioural intervention on employment-related outcomes for individuals with mood and anxiety disorders. Cognitive Behaviour Therapy, 37, 247-251. doi: 10.1080/16506070802473189

Krause, N., Dasinger, L. K., \& Neuhauser, F. (1998). Modified work and return to work: A review of the literature. Journal of Occupational Rehabilitation, 8, 113-139. doi:10.1023/A:1023015622987

Lagerveld, S. E., Blonk, R. W. B., Brenninkmeijer, V., \& Schaufeli, W. B. (2010a). Return to work among employees with mental health problems: 
Development and validation of a self-efficacy questionnaire. Work \& Stress, 24, 359-375. doi:10.1080/02678373.2010.532644

Lagerveld, S. E., Bültmann, U., Franche, R. L., van Dijk, D. J. H., Vlasveld, M. C., van der Feltz-Cornelis, C. M., ... Nieuwenhuijsen, K. (2010b). Factors associated with work participation and work functioning in depressed workers: A systematic review. Journal of Occupational Rehabilitation, 20, 275-292. doi:10.1007/s10926-009-9224-x

Lambeek, L. C., van Mechelen, W., Knol, D. L., Loisel, P., \& Anema, J. R. (2010). Integrated care for chronic back pain: A randomized controlled trial evaluating a systems approach to reduce disability in working and private life. British Medical Journal, 340, c1035. doi:10.1136/ bmj.c1035

Lander, F., Firche, C., Tornemand, H., Andersen, J. H., \& Kirkeskov, L. (2009). Can we enhance the ability to return to work among workers with stress-related disorders? BMC Public Health, 9, 372-377. doi: 10.1186/1471-2458-9-372

Little, T. D., Schnabel, K. U., \& Baumert, J. (2000). Modeling longitudinal and multilevel data: Practical issues, applied approaches, and specific examples. Mahwah, NJ: Erlbaum.

Loisel, P., Abenhaim, L., Durand, P., Esdaile, J. M., Suissa, S., Gosselin, L., . . Lemaire, J. (1997). A population-based, randomized clinical trial on back pain management. Spine, 22, 2911-2918. doi:10.1097/ 00007632-199712150-00014

Longmore, R., \& Worrell, M. (2007). Do we need to challenge thoughts in Cognitive Behavioural Therapy? Clinical Psychology review, 27, 173187. doi:10.1016/j.cpr.2006.08.001

Lovibond, S. H., \& Lovibond, P. F. (1995). Manual for the Depression Anxiety Stress Scales. Sydney, Australia: The Psychology Foundation of Australia.

Meichenbaum, D., \& Cameron, R. (1982). Cognitive behavior therapy. In G. T. Wilson \& C. M. Franks (Eds.), Contemporary behavior therapy: Conceptual and empirical foundations (pp. 310-338). New York, NY: Guilford.

Myette, T. L. (2008). Research on depression in the workplace: Where do we go from here? Journal of Occupational and Environmental Medicine, 50, 492-500. doi:10.1097/JOM.0b013e31816f855a

Nieuwenhuijsen, K. (2004). Employees with common mental disorders: From diagnosis to return to work (Dissertation). University of Amsterdam, the Netherlands.

Nieuwenhuijsen, K., Bultmann, U., Neumeyer-Gromen, A., Verhoeven, A. C., Verbeek, J. H., \& van der Feltz-Cornelis, C. M. (2008). Interventions to improve occupational health in depressed people. Cochrane Database Systematic Reviews, 2, CD006237.

Noordik, E., van Dijk, F. J., Nieuwenhuijsen, K., \& van der Klink, J. J. L. (2009). Effectiveness and cost-effectiveness of an exposure-based return-to-work programme for patients on sick leave due to common mental disorders: Design of a cluster-randomized controlled trial. $B M C$ Public Health, 9, 140. doi:10.1186/1471-2458-9-140

Nystuen, P., Hagen, K. B., \& Herrin, J. (2006). Solution-focused intervention for sick listed employees with psychological problems or muscle skeletal pain: A randomised controlled trial. BMC Public Health, 6, 69. doi:10.1186/1471-2458-6-69

Plaisier, I. (2009). Work and mental health. Studies on the impact of job characteristics, social roles and gender (Dissertation). Free University Amsterdam, The Netherlands.

Price, R. H., van Ryn, M., \& Vinokur, A. D. (2002). Impact of a preventive job search intervention on the likelihood of depression among the unemployed. Journal of Health and Social Behavior, 33, 158-167. doi: $10.2307 / 2137253$

Rai, D., Skapinakis, P., Wiles, N., Lewis, G., \& Araya, R. (2010). Common mental disorders, subthreshold symptoms and disability: Longitudinal study. The British Journal of Psychiatry, 197, 411-412. doi:10.1192/ bjp.bp. 110.079244

Rasbash, J., Browne, W., Goldstein, H., Yang, M., Plewis, I., \& Healy, M.
(2000). A user's guide to MLwiN. London, UK: University of London, Multilevel Models Project.

Rebergen, D. S. (2009). Effectiveness of guideline-based care of workers with mental health problems (Dissertation). Free University Amsterdam, The Netherlands.

Sanderson, K., \& Andrews, G. (2006). Common mental disorders in the workforce: Recent findings from descriptive and social epidemiology. Canadian Journal of Psychiatry, 51, 63-75.

Schaufeli, W. B., \& van Dierendonck, D. (2000). Handleiding van de Utrechtse Burnout Schaal (UBOS) [Manual of the Utrecht Burnout Scale]. Lisse, The Netherlands: Swets \& Zeitlinger.

Schene, A. H., Koeter, M. W. J., Kikkert, M. J., Swinkels, J. A., \& McCrone, P. (2007). Adjuvant occupational therapy for work-related major depression works: Randomized trial including economic evaluation. Psychological Medicine, 37, 351-362. doi:10.1017/ S0033291706009366

Seymour, L., \& Grove, B. (2005). Workplace interventions for people with common mental health problems: A systematic review. London, UK: British Occupational Health Research Foundation.

Stress Impact (2006). Integrated report of Stress Impact: On the impact of changing social structures on stress and quality of life: Individual and social perspectives. Retrieved from www.surrey.ac.uk/Psychology/ stress-impact/publications

ten Have, M., de Graaf, R., Vollebergh, W., \& Beekman, A. (2004). What depressive symptoms are associated with the use of care services? Results from the Netherlands Mental Health Survey and Incidence Study (NEMESIS). Journal of Affective Disorders, 80, 239-248. doi:10.1016/ S0165-0327(03)00132-0

UWV (2007).Quarterly Report Knowledge Center UWV 2007-III. Retrieved from www.uwv.nl/Images/UKV\%202007-III_tcm26147000.pdf

van der Bossche, S., \& Houtman, I. (2007). Longitudinal quantitative study on long term absent employees: The Netherlands. Stress Impact Report. Retrieved from www.surrey.ac.uk/Psychology/stressimpact/ publications/wp5

van der Klink, J. J. L., Blonk, R. W. B., Schene, A. H., \& van Dijk, F. J. (2003). Reducing long term sickness absence by an activating intervention in adjustment disorders: A cluster randomised controlled design. Occupational \& Environmental Medicine, 60, 429-437. doi:10.1136/ oem.60.6.429

van Oostrom, S. H., van Mechelen, W., Terluin, B., de Vet, H. C. W., Knol, D. L., \& Anema, J. R. (2010). A workplace intervention for sick-listed employees with distress: Results of a randomised controlled trial. Occupational and Environmental Medicine, 67, 596-602. doi:10.1136/ oem.2009.050849

van Rhenen, W., Van Dijk, F., Schaufeli, W. B., \& Blonk, R. W. B. (2008), Coping and sickness absence. International Archives for Occupational and Environmental, 81, 461-472. doi:10.1007/s00420-007-0238-4

van Schie, J., Blonk, R. W. B., \& Lagerveld, S. E. (2005). Werkhervatting bij arbeidsgerelateerde psychische klachten, behandelingsprotocol [Return to Work and work-related psychological complaints, treatment protocol]. Hoofddorp, The Netherlands: TNO.

Vlasveld, M. C., Anema, J. R., Beekman, T. F., van Mechelen, W., Hoedeman, R., van Marwijk, H. W. J., . . van der Feltz-Cornelis, C. M. (2008). Multidisciplinary collaborative care for depressive disorder in the occupational health setting: Design of a randomised controlled trial and cost-effectiveness study. BMC Health Services Research, 8, 99. doi:10.1186/1472-6963-8-99

Wang, J. L., Adair, C. E., \& Patten, S. B. (2006). Mental health and related disability among workers: A population-based study. American Journal of Industrial Medicine, 49, 514-522. doi:10.1002/ajim.20319

Wasiak, R., Young, A. E., Roessler, R. T., McPherson, K. M., van Poppel, M. N., \& Anema, J. R. (2007). Measuring return to work. Journal of 
Occupational Rehabilitation, 17, 766-781. doi:10.1007/s10926-0079101-4

Westen, D., Novotny, C. M., \& Thompson-Brenner, H. (2004). The empirical status of empirically supported psychotherapies: Assumptions, findings, and reporting in controlled clinical trials. Psychological Bulletin, 130, 631-663. doi:10.1037/0033-2909.130.4.631

WHO (2002). The World Health Report. Mental Health: New Understanding, New hope. Geneva, Switzerland: World Health Organization.
Wright, J. H. (2006). Cognitive behavior therapy: Basic principles and recent advances. Focus, 4, 173-178.

Received December 14, 2010 Revision received November 15, 2011 Accepted December 6, 2011

\section{Correction to Odle-Dusseau, Britt, and Green-Shortridge (2012)}

In the article, "Organizational Work-Family Resources as Predictors of Job Performance and Attitudes: The Process of Work-Family Conflict and Enrichment," by Heather N. Odle-Dusseau, Thomas W. Britt, and Tiffany M. Green-Shortridge (Journal of Occupational Health Psychology, 2012, Vol. 17, No. 1, pp. 28-40), the following references contained spelling errors:

Kossek, E. E., \& Nicol, V. (1992). The effects of on-site child care on employee attitudes and performance. Personnel Psychology, 45, 485-509. doi:10.1111/j.1744-6570.1992.tb00857.x Kossek, E. E., Pichler, S., Bodner, T., \& Hammer, L. B. (2011). Workplace social support and work-family conflict: A meta-analysis clarifying the influence of general and work-familyspecific supervisor and organizational support. Personnel Psychology, 64, 289-313. doi:10.1111/ j.1744-6570.2011.01211.x

The online version of this article has been corrected.

DOI: $10.1037 / \mathrm{a} 0027138$ 